\title{
Use of Single Crystal Masks for Improved Mill Characteristics in High Current Xenon Plasma FIB instrumentation
}

\author{
Srinivas Subramaniam ${ }^{1}$, Linda Smath ${ }^{1}$, Adriane Brown ${ }^{1}$ and Kevin Johnson ${ }^{1}$. \\ ${ }^{1 .}$ Intel Corporation, Hillsboro, OR, U.S.A.
}

Technology development activities in the semiconductor industry face multiple challenges in scaling and velocity with the forward progression of each technology node. Despite increased complexities in newer process generations, reducing throughput time (TPT) for failure analysis (FA) and development remains a priority.

High current focused ion beam tools are well placed to support development, and reduce TPT constraints. While these tools possess significant advantages, considerable efforts are underway to understand and optimize the newer technologies. Additionally, the operational performance of Xenon Plasma FIB tools is limited by their large probe's characteristics, which include curtaining and degradations of milled regions and mediocre final quality of the cross section surface. We have, in our earlier work, looked at understanding the intrinsic design and operational aspects of Xenon Plasma FIBs with the aim of improving performance. This paper will look at an external performance improvement approach [1,2]. We have developed a novel single crystal mask process to optimize the tool for applications in semiconductor failure analysis, which significantly improves performance and matches results from conventional Gabased ion beam systems while retaining the inherent high current TPT benefits of the Xenon Plasma FIB.

Conventional I/E-Beam capping and masking layers are used extensively for FA and development activities to preserve and protect specimens during initial preparation. However masks deposited using these techniques tend to have poor material properties associated with the low temperature deposition processes, such as porosity, impurities and poor crystallinity that affect the physical and chemical properties and performance of the films [3]. Our novel approach uses a single crystal mask that has been fabricated and attached to the specimen prior to the milling operation. Once positioned, the mask enables multiple functions, acting as a sacrificial layer while utilizing the single crystal surface to tailor the beam, minimizing artifacts, and obtaining surface finishes matched to conventional gallium milling methods. The straightforward implementation, along with ease of removal of the mask, allows the single crystal mask to extend itself to multiple application scenarios in FA activities.

A Tescan Fera Xenon Plasma High Current Dual Beam Instrument was used in these experiments. This tool uses a $90^{\circ}$ offset rotation mill technique at a $35^{\circ}$ tilt for the bulk material removal followed by normal milling and polishing at $55^{\circ}$ to obtain the best final inspection conditions. SEM imaging in the secondary electron mode (Vac 10KV) was used to image the samples, ensuring good topographic contrast at the milled regions. Figure 1 shows as sample that has been milled using a conventional GIS deposited tungsten film. The conventionally milled sample shows poor surface finish characteristics due to curtaining and beam tails resulting from a recessed top surface. Excessive curtaining can also be seen to interfere with the finer feature details. Figure 2 shows a milled region made using the single crystal mask. The sample milled using the single crystal mask shows a relatively curtain free surface with no recess and lesser curtaining being observed. 


\section{References:}

[1] Subramaniam et al, Microscopy and Microanalysis (2014) p. 296.

[2] Subramaniam, et al, Microscopy and Microanalysis (2015) p. 1841.

[3] Kim et. al, J. Mater. Res., 14 (1999), p. 634.

Figure 1 SE image of final surface on conventional Tungsten GIS capped sample after milling.

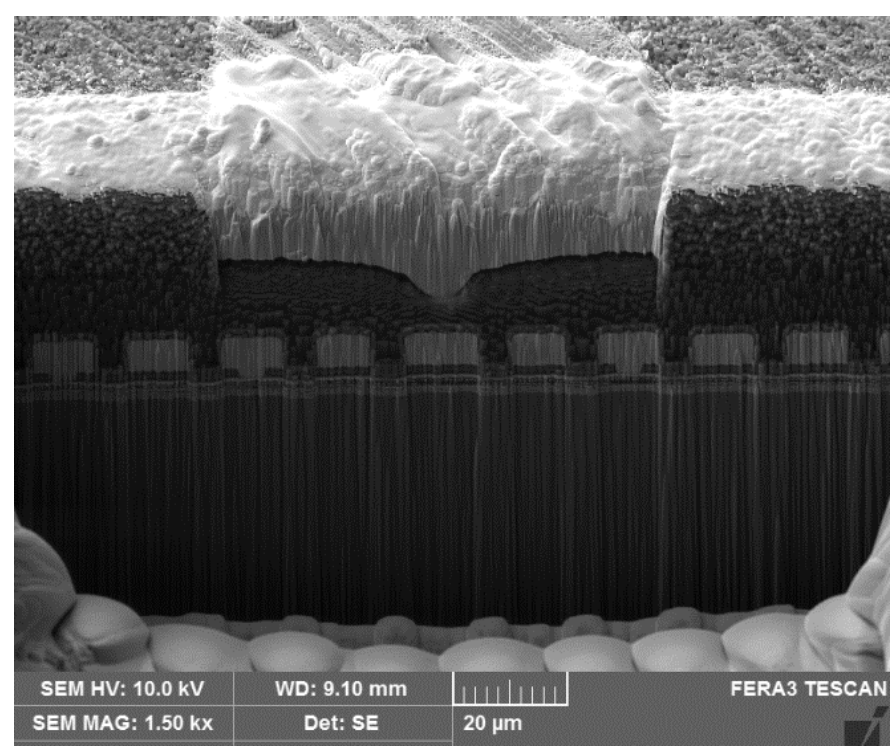

Figure 2 SE image of final surface on sample which has been prepared with a single crystal mask prior to milling.

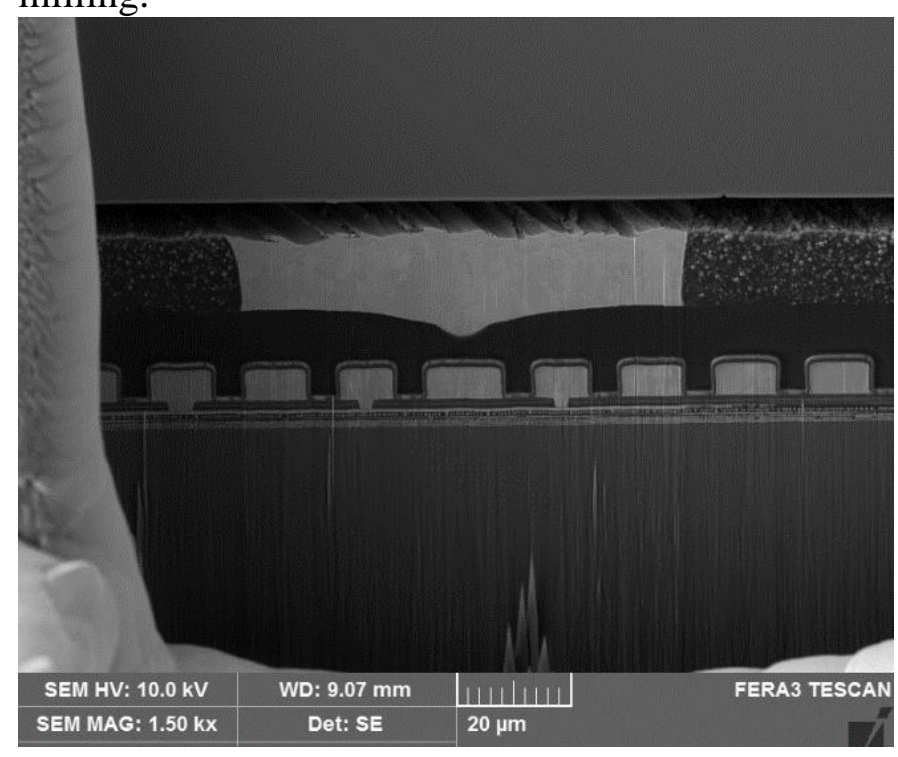

